\title{
The Casimir effect as scattering problem
}

\author{
A Wirzba \\ Institut für Kernphysik, Forschungszentrum Jülich, D-52425 Jülich, Germany \\ E-mail: a.wirzba@fz-juelich.de
}

\begin{abstract}
We show that Casimir-force calculations for a finite number of nonoverlapping obstacles can be mapped onto quantum-mechanical billiard-type problems which are characterized by the scattering of a fictitious point particle off the very same obstacles. With the help of a modified Krein trace formula the genuine/finite part of the Casimir energy is determined as the energy-weighted integral over the log-determinant of the multi-scattering matrix of the analog billiard problem. The formalism is self-regulating and inherently shows that the Casimir energy is governed by the infrared end of the multi-scattering phase shifts or spectrum of the fluctuating field. The calculation is exact and in principle applicable for any separation(s) between the obstacles. In practice, it is more suited for large- to medium-range separations. We report especially about the Casimir energy of a fluctuating massless scalar field between two spheres or a sphere and a plate under Dirichlet and Neumann boundary conditions. But the formalism can easily be extended to any number of spheres and/or planes in three or arbitrary dimensions, with a variety of boundary conditions or nonoverlapping potentials/non-ideal reflectors.
\end{abstract}

PACS numbers: 03.65.Nk, 03.65.Sq, 03.70.+k, 05.45.Mt

\section{Introduction}

In 1948, the Dutch physicist H B G Casimir predicted the remarkable effect [1] that two parallel, very closely spaced, uncharged metallic plates attract each other in vacuum. The origin of this force can be traced back to the modifications of the zero-point fluctuations of the electromagnetic field by the presence of the two plates (at distance $L$ ) relative to the free case, or rather, relative to the case where the plates are separated by an infinite distance. Mathematically, this corresponds to the following difference of two mode sums:

$$
\left.\sum \frac{1}{2} \hbar \omega_{k}\right|_{\operatorname{plates}(L)}-\left.\sum \frac{1}{2} \hbar \omega_{k}\right|_{\operatorname{plates}(L \rightarrow \infty)} .
$$

The distinctive feature of the Casimir effect is that it depends on the geometry in a non-intuitive way: its strength and, perhaps, its sign are geometry-dependent (for a review see [2]).

Let us look at the other side of the 'coin' and take the geometry dependence as guiding principle for the construction of the Casimir effect; i.e. we will define the 
Casimir energy as that part of the vacuum energy that results from the geometrydependent and therefore changeable part of the pertinent density of states. In fact, in Casimir-type calculations, the density of states $\rho(E)=\sum_{E_{k}} \delta\left(E-E_{k}\right)$, where $\left\{E_{k}\right\}$ are the eigenenergies of the modes, can be split into three parts:

$$
\rho(E)=\rho_{0}(E)+\rho_{\text {bulk }}(E)+\delta \rho_{\mathrm{C}}(E) .
$$

(i) There is a free part $\rho_{0}(E)$ over the homogeneous part of the background (the fluctuating field, matter fields etc.) that is completely unaffected by the presence of the obstacles; (ii) the bulk part $\rho_{\text {bulk }}(E)$ sums up the modifications of the spectrum (excluded volumes and surface contributions including Friedel oscillations) by each of the individual obstacles as though they were still infinitely separated; (iii) finally the remaining part $\delta \rho_{\mathrm{C}}(E)$ is the genuinely geometry-dependent part of the density of states which 'knows' about the relative separations and angles between the various obstacles. It is the latter that determines the Casimir energy when it is integrated up, weighted by the energy $E$ :

$$
\mathcal{E}_{\mathrm{C}} \equiv \frac{1}{2} \int_{0}^{\infty} \mathrm{d} E E \delta \rho_{\mathrm{C}}(E)=-\frac{1}{2} \int_{0}^{\infty} \mathrm{d} E \mathcal{N}_{\mathrm{C}}(E),
$$

where $\mathcal{N}_{\mathrm{C}}(E)=\int_{0}^{E} \mathrm{~d} E^{\prime} \delta \rho_{\mathrm{C}}\left(E^{\prime}\right)$ is the geometry-dependent part of the integrated density of states (or number of states),

$$
\mathcal{N}(E) \equiv \int_{0}^{E} \mathrm{~d} E^{\prime} \rho\left(E^{\prime}\right)=\sum_{E_{k}} \Theta\left(E-E_{k}\right) .
$$

Note that the integrals in (2) do not converge absolutely, but only conditionally because of the oscillating behaviour of $\delta \rho_{\mathrm{C}}(E)$ and $\mathcal{N}_{\mathrm{C}}(E)$. The inclusion of e.g. an exponential damping factor (compare with the analogous tilt of the integration path(s) in (13)), which can be removed after the integration(s) are performed, cures this problem and also eliminates the spurious upper-boundary contribution $\left.\frac{1}{2} E \mathcal{N}_{\mathrm{C}}(E)\right|^{\infty}$ from the partial integration. The lower boundary term $\left.\frac{1}{2} E \mathcal{N}_{\mathrm{C}}(E)\right|_{0}$ vanishes automatically.

\section{The mapping of the Casimir calculation onto a scattering problem}

The Casimir calculation for two parallel plates at distance $L$ was simple since the problem is separable, i.e. quasi-one-dimensional if the plates had infinite extent. For more complicated geometries than two parallel plates only the proximity force approximation [3] is left in general (see, however, section 6).

However, as shown in [4, 5] for the non-relativistic fermionic Casimir effect and in [6. 7] for the scalar Casimir effect t, the Casimir energy between any finite number of non-overlapping spherical obstacles, i.e. spheres and cylinders in 3 dimensions or disks in 2 dimensions, can be solved exactly, although the corresponding quantum-mechanical problems are not separable any longer. In fact, these calculations simplify because of

$\mp$ In the scalar Casimir effect the fluctuating field is not the electromagnetic one, but a massless scalar one with the dispersion $E=\hbar c|\vec{k}|$. In the non-relativistic fermionic Casimir effect, the background does not consist of fluctuating fields, but of (a Fermi sea of) non-relativistic fermionic matter waves. 
Krein's trace formula [8] which maps - under the condition that the potentials of the obstacles are sufficiently short-ranged - the difference in the total level densities - with and without obstacles - to the energy variation of the total phase shift\&. For the special case of $n$ spherically symmetric obstacles of radii $a_{i}$ at mutual center-to-center separation $r_{i j}>a_{i}+a_{j}$ (such that they do not overlap) and mutual angles $\alpha_{i j}$ the Krein formula reads

$$
\begin{aligned}
\delta \bar{\rho}(E)=\bar{\rho}(E)-\bar{\rho}_{0}(E) & =\frac{1}{2 \pi \mathrm{i}} \frac{\mathrm{d}}{\mathrm{d} E} \operatorname{tr} \ln S_{n}\left(E,\left\{a_{i}\right\},\left\{\vec{r}_{i j}\right\}\right) \\
& =\frac{1}{2 \pi \mathrm{i}} \frac{\mathrm{d}}{\mathrm{d} E} \ln \operatorname{det} S_{n}\left(E,\left\{a_{i}\right\},\left\{\vec{r}_{i j}\right\}\right),
\end{aligned}
$$

where the distances $r_{i j}$ and angles $\alpha_{i j}$ have been combined to separation vectors $\vec{r}_{i j}$ and where $i, j=1,2, \cdots, n$ label the obstacles. Note that the level densities on the left hand side are averaged over an energy-interval larger than the mean-level spacing in the volume $V$ of the entire system which will be taken to infinity in the end. This averaging is done in order to match to the right hand side which is defined in terms of the total $S$-matrix $S_{n}\left(E,\left\{a_{i}\right\},\left\{\vec{r}_{i j}\right\}\right)$ of the $n$-disk system in 2 dimensions [10, 11, 12] or the $n$ sphere system in 3 dimensions [13, respectively, and which is therefore continuous. In fact, in [11, 12] it was shown that the determinant of $n$-disk S-matrix is finite (except at the known poles and branch points), although the matrix has an infinite number of components, since the pertinent T-matrix was proved to be trace class. The 3dimensional $n$-sphere case was discussed in [13]. In this way the Casimir calculation for a finite number of non-overlapping obstacles is mapped onto the quantum-mechanical analog of a classical billiard-type problem which is characterized by the scattering of a fictitious point particle off the very same obstacles. For the case of only one spherical obstacle the scattering problem is separable, but uninteresting as there is no Casimir effect. For two spherical obstacles the scattering problem is non-separable and classically hyperbolic, and for more than two obstacles it is in general even classically chaotic [14, 10, 15, 16, 17].

Let us remark that the energy-averaging and the infinite volume limit do not commute, but that first the volume has to be taken to infinity and only then the averaging interval can go to zero. This is obvious if one takes semiclassical considerations into account where the determinant over the $n$-sphere/disk matrix is given in terms of periodic orbits and possible Weyl term corrections, see [11, 12]. For this purpose let us put the scattering system at the middle of a large container which can be taken, without loss of generality, to be spherical of finite radius $R$ and consider this container together with its empty reference container that is of equal size. Now, as long as the containers have a finite size, there exist three classes of periodic orbits. (i) The generic scattering periodic orbits that only bounce between the obstacles belonging to the scattering system and that semiclassically specify the determinant of the scattering matrix. (ii) A first class of spurious periodic orbits which solely are there because of the container and which only bounce between the walls of the container. The subtraction of $\S$ See [9] for a precursor of Krein's formula for potentials with spherical symmetry. 
the reference system, in fact, removes this class of spurious orbits. (iii) A second class of spurious periodic orbits which bounce between the container walls and the scattering obstacles and which do not exist in the empty reference container. As the length $L_{\text {p.o. }}$ of these periodic orbits will increase with the size of the container, their contribution to the periodic orbit sum can be suppressed if a small imaginary term i $\epsilon$ is added to the energy or wave number $k \equiv|\vec{k}|$ which is here the same because the dispersion is $E=\hbar c|\vec{k}|$. The phase of the orbit acquires therefore an exponential damping factor

$$
\mathrm{e}^{\mathrm{i} k L_{\mathrm{p} . \mathrm{o}}} \rightarrow \mathrm{e}^{\mathrm{i}(k+\mathrm{i} \epsilon) L_{\text {p.o }}}
$$

which smooths (averages) the total and background densities of states in the Krein formula

$$
\lim _{\epsilon \rightarrow 0_{+}} \lim _{R \rightarrow \infty}\left\{\rho(k+\mathrm{i} \epsilon, R)-\rho_{0}(k+\mathrm{i} \epsilon, R)\right\}=\frac{1}{2 \pi \mathrm{i}} \frac{\mathrm{d}}{\mathrm{d} E} \operatorname{tr} \ln S_{n}\left(E(k),\left\{a_{i}\right\},\left\{\vec{r}_{i j}\right\}\right) .
$$

This explains the smoothing/averaging procedure in (44).

\section{The mapping to the multi-scattering matrix}

In the following, we will show that the geometry-dependent Casimir fluctuations can be extracted from the multiple-scattering part of the S-matrix. For this purpose let us once more explain the philosophy: (i) we start out with a large, but finite container of radius $R$ which will be filled with the background field (here the massless scalar field or a fermionic matter field) and which eventually will be taken to infinite size. The total density of states is therefore equal to the background density: $\rho(k+\mathrm{i} \epsilon, R)=\rho_{0}(k+\mathrm{i} \epsilon, R)$. A suppression term is added to the wave number $k$ as explained in the last section. (ii) Next we will also put the spherical obstacles of radii $a_{i}$ into the large container, where we make sure that the mutual separation $r_{i j}$ between any pair of obstacles is large, i.e. comparable with the size of the container. The total density of states has to be readjusted because the background field is affected by excluded volumes due to the obstacles and by boundary effects (including Friedel oscillations) due to the imposed boundary conditions at the surface of the obstacles, say Dirichlet boundary conditions:

$$
\rho\left(k+\mathrm{i} \epsilon, R,\left\{a_{i}\right\}\right)=\rho_{0}(k+\mathrm{i} \epsilon, R)+\sum_{i=1}^{n} \underbrace{\rho_{\mathrm{W}}\left(k+\mathrm{i} \epsilon, R, a_{i}\right)}_{\text {Weyl term }} .
$$

(iii) Now, the obstacles are moved close to each other such that the actual system of a finite number of non-overlapping spherical obstacles is formed. Again the density of states has to be readjusted, this time by the geometry-dependent term $\delta \rho_{\mathrm{C}}$ which depends on all radii $\left\{a_{i}\right\}$ and on all separation vectors $\left\{\vec{r}_{i j}\right\}$ :

$$
\begin{aligned}
\rho\left(k+\mathrm{i} \epsilon, R,\left\{a_{i}\right\},\left\{\vec{r}_{i j}\right\}\right)= & \rho_{0}(k+\mathrm{i} \epsilon, R)+\sum_{i=1}^{n} \rho_{\mathrm{W}}\left(k+\mathrm{i} \epsilon, R, a_{i}\right) \\
& +\delta \rho_{\mathrm{C}}\left(k+\mathrm{i} \epsilon, R,\left\{a_{i}\right\},\left\{\vec{r}_{i j}\right\}\right) .
\end{aligned}
$$

(iv) By inserting the difference of (77) and the background density of states $\rho_{0}$ into the Krein formula and by taking the double $\operatorname{limit}_{\lim _{\epsilon \rightarrow 0_{+}}} \lim _{R \rightarrow \infty}$ we effectively remove the 
container walls and map the problem to the scattering system:

$$
\begin{aligned}
& \lim _{\epsilon \rightarrow 0_{+}} \lim _{R \rightarrow \infty}\left\{\rho\left(\left(k+\mathrm{i} \epsilon, R,\left\{a_{i}\right\},\left\{\vec{r}_{i j}\right\}\right)-\rho_{0}(k+\mathrm{i} \epsilon, R)\right\}\right. \\
& \equiv \bar{\rho}\left(k,\left\{a_{i}\right\},\left\{\vec{r}_{i j}\right\}\right)-\bar{\rho}_{0}(k)=\frac{1}{2 \pi \mathrm{i}} \frac{\mathrm{d}}{\mathrm{d} k} \ln \operatorname{det} S_{n}\left(k,\left\{a_{i}\right\},\left\{\vec{r}_{i j}\right\}\right),
\end{aligned}
$$

where $\ln \operatorname{det} S_{n}\left(k,\left\{a_{i}\right\},\left\{\vec{r}_{i j}\right\}\right)$ is the total phase shift expressed through the $n$ sphere/disk S-matrix $S_{n}\left(k,\left\{a_{i}\right\},\left\{\vec{r}_{i j}\right\}\right)$. As shown in [11, 12] and [13], respectively, the determinant of the $n$-disk/sphere S-matrix separates into a product of the determinants of the 1 -disk/sphere S-matrices $S_{1}\left(E, a_{i}\right)$, where $a_{i}$ is the radius of the single scatterer $i$, and the ratio of the determinant of the inverse multi-scattering matrix $M$ and its complex conjugate:

$$
\operatorname{det} S_{n}\left(k,\left\{a_{i}\right\},\left\{\vec{r}_{i j}\right\}\right)=\left\{\prod_{i=1}^{n} \operatorname{det} S_{1}\left(k, a_{i}\right)\right\} \frac{\operatorname{det}\left(M\left(k^{*},\left\{a_{i}\right\},\left\{\vec{r}_{i j}\right\}\right)^{\dagger}\right)}{\operatorname{det} M\left(k,\left\{a_{i}\right\},\left\{\vec{r}_{i j}\right\}\right)} .
$$

Note that each and every determinant in (9) is finite and exists separately, since the pertinent T-matrices are all trace class [11, 12, 13].

When inserted into Krein's formula, the product over the single-scatterer determinants generates just the bulk (or Weyl term) contribution to the density of states

$$
\begin{aligned}
& \lim _{\epsilon \rightarrow 0_{+}} \lim _{R \rightarrow \infty} \rho_{\text {bulk }}\left(k+\mathrm{i} \epsilon, R,\left\{a_{i}\right\}\right) \equiv \sum_{i=1}^{n} \lim _{\epsilon \rightarrow 0_{+}} \lim _{R \rightarrow \infty} \rho_{\mathrm{W}}\left(k+\mathrm{i} \epsilon, R, a_{i}\right) \\
& \equiv \sum_{i=1}^{n} \bar{\rho}_{\mathrm{W}}\left(k, a_{i}\right)=\frac{1}{2 \pi \mathrm{i}} \frac{\mathrm{d}}{\mathrm{d} k} \sum_{i=1}^{n} \ln \operatorname{det} S_{1}\left(k, a_{i}\right),
\end{aligned}
$$

which takes care of the excluded volume terms and the surface terms (including Friedel oscillations). The geometry-dependent part of the density of states

$$
\delta \bar{\rho}_{\mathrm{C}}\left(k,\left\{a_{i}\right\},\left\{\vec{r}_{i j}\right\}\right) \equiv \lim _{\epsilon \rightarrow 0_{+}} \lim _{R \rightarrow \infty} \delta \rho_{\mathrm{C}}\left(k+\mathrm{i} \epsilon, R,\left\{a_{i}\right\},\left\{\vec{r}_{i j}\right\}\right)
$$

is therefore given by a modified Krein equation [4] which is formulated in terms of the inverse multi-scattering matrix $M\left(k,\left\{a_{i}\right\},\left\{\vec{r}_{i j}\right\}\right)$ instead of the full S-matrix

$$
\begin{aligned}
\delta \bar{\rho}_{\mathrm{C}}\left(k,\left\{a_{i}\right\},\left\{\vec{r}_{i j}\right\}\right) & =\bar{\rho}\left(k,\left\{a_{i}\right\},\left\{\vec{r}_{i j}\right\}\right)-\bar{\rho}_{0}(k)-\sum_{i=1}^{n} \bar{\rho}_{\text {Weyl }}\left(k, a_{i}\right) \\
& =-\frac{1}{\pi} \operatorname{Im} \frac{\mathrm{d}}{\mathrm{d} k} \ln \operatorname{det} M\left(k,\left\{a_{i}\right\},\left\{\vec{r}_{i j}\right\}\right) .
\end{aligned}
$$

Note that in this expression all the possibly divergent terms connected with the sharpsurface limits are subtracted out. In fact, by mapping the system to a multi-scattering problem the calculation is self-regulating: an additional regulator is not needed (except the energy-damping mentioned below (2) which can be removed in the end, see also [6]).

The pertinent Casimir energy can then be read off from the energy-weighted (or wave-number-weighted) integral

$$
\begin{aligned}
\mathcal{E}_{\mathrm{C}} & =\int_{0}^{\infty} \mathrm{d} E \frac{1}{2} E \delta \bar{\rho}_{C}\left(E,\left\{a_{i}\right\},\left\{\vec{r}_{i j}\right\}\right)=\frac{1}{2} \hbar c \int_{0}^{\infty} \mathrm{d} k k \delta \bar{\rho}_{C}\left(k,\left\{a_{i}\right\},\left\{\vec{r}_{i j}\right\}\right) \\
& =-\frac{1}{2} \hbar c \int_{0}^{\infty} \mathrm{d} k \overline{\mathcal{N}}_{\mathrm{C}}\left(k,\left\{a_{i}\right\},\left\{\vec{r}_{i j}\right\}\right)=\frac{\hbar c}{2 \pi} \int_{0}^{\infty} \mathrm{d} k \operatorname{Im} \ln \operatorname{det} M(k)
\end{aligned}
$$


The Casimir effect as scattering problem

$$
\begin{aligned}
& =\frac{\hbar c}{4 \pi \mathrm{i}}\left[\int_{0}^{\infty\left(1+\mathrm{i} 0_{+}\right)} \mathrm{d} k \ln \operatorname{det} M(k)-\int_{0}^{\infty\left(1-\mathrm{i} 0_{+}\right)} \mathrm{d} k \ln \operatorname{det} M\left(k^{*}\right)^{\dagger}\right] \\
& =\frac{\hbar c}{2 \pi} \int_{0}^{\infty} \mathrm{d} k_{4} \ln \operatorname{det} M\left(\mathrm{i} k_{4}\right),
\end{aligned}
$$

where the dependences on the radii and separation vectors were suppressed in the argument of the inverse multi-scattering matrix $M(k) \equiv M\left(k,\left\{a_{i}\right\},\left\{\vec{r}_{i j}\right\}\right)$ for simplicity. In the last step a Wick rotation $k \rightarrow \mathrm{i} k_{4}$ was performed in the first, and $k \rightarrow-\mathrm{i} k_{4}$ in the second integral, and the relation $\operatorname{det} M\left(\mathrm{i} k_{4}\right)=\operatorname{det} M\left(\mathrm{i} k_{4}\right)^{\dagger}$ was applied which follows from the relation $\operatorname{det} M(k)=\operatorname{det} M\left((-k)^{*}\right)^{\dagger}[6]$. As a corollary note that

$$
\begin{aligned}
& \frac{\hbar c}{2 \pi} \int_{0}^{\infty} \mathrm{d} k k^{2 n+1} \mathrm{Im} \ln \operatorname{det} M(k) \\
& =\mathrm{i}(-1)^{n} \frac{\hbar c}{4 \pi} \int_{0}^{\infty} \mathrm{d} k_{4} k_{4}^{2 n+1}\left[\ln \operatorname{det} M\left(\mathrm{i} k_{4}\right)-\ln \operatorname{det} M\left(\mathrm{i} k_{4}\right)^{\dagger}\right]=0,
\end{aligned}
$$

e.g. the Casimir energy over modes with a non-relativistic dispersion $E=\hbar^{2} k^{2} / 2 m_{\mathrm{N}}$, where $m_{\mathrm{N}}$ is the pertinent mass, integrates to zero [6], unless there is a finite upper cutoff, as e.g. the Fermi momentum $k_{\mathrm{F}}$ in the case of the so-called fermionic Casimir effect [4].

Note that the final expression (13) for the Casimir energy is obviously finite, since $\operatorname{det} M\left(\mathrm{i} k_{4}\right)$ vanishes rapidly with increasing value of $k_{4}$, and moreover, for the same reason, it is dominated by the infrared end of the integration.

\section{The multi-scattering matrix and the fermionic Casimir effect}

The modified Krein equation (12) is especially useful as there there exists a close-form expression for the inverse multi-scattering matrix for $n$ spheres (of radii $a_{j}$ and mutual distances $r_{j j^{\prime}}$, where the indices $j, j^{\prime}=1,2, \cdots, n$ label the spheres) in terms of spherical Bessel and Hankel functions of first kind, spherical harmonics and 3j-symbols [13]:

$$
\begin{aligned}
& M_{l m, l^{\prime} m^{\prime}}^{j j^{\prime}}=\delta^{j j^{\prime}} \delta_{l l^{\prime}} \delta_{m m^{\prime}}+\left(1-\delta^{j j^{\prime}}\right) \mathrm{i}^{2 m+l^{\prime}-l} \sqrt{4 \pi(2 l+1)\left(2 l^{\prime}+1\right)} \\
& \quad \times\left(\frac{a_{j}}{a_{j^{\prime}}}\right)^{2} \frac{j_{l}\left(k a_{j}\right)}{h_{l^{\prime}}^{(1)}\left(k a_{j^{\prime}}\right)} \sum_{l^{\prime \prime}=0}^{\infty} \sum_{m^{\prime \prime}=-l^{\prime}}^{l^{\prime}} \sqrt{2 l^{\prime \prime}+1} \mathrm{i}^{l^{\prime \prime}}\left(\begin{array}{ccc}
l^{\prime \prime} & l^{\prime} & l \\
0 & 0 & 0
\end{array}\right) \\
& \quad \times\left(\begin{array}{ccc}
l^{\prime \prime} & l^{\prime} & l \\
m-m^{\prime \prime} & m^{\prime \prime} & -m
\end{array}\right) D_{m^{\prime}, m^{\prime \prime}}^{l^{\prime}}\left(j, j^{\prime}\right) h_{l^{\prime \prime}}^{(1)}\left(k r_{j j^{\prime}}\right) Y_{l^{\prime \prime}}^{m-m^{\prime \prime}}\left(\hat{r}_{j j^{\prime}}^{(j)}\right) .
\end{aligned}
$$

Here $l, l^{\prime}$ and $m, m^{\prime}$ are total angular momentum and associated magnetic quantum numbers, respectively. The rotation matrix $D_{m^{\prime}, m^{\prime \prime}}^{l^{\prime}}\left(j, j^{\prime}\right)$ maps the local coordinate system of the sphere $j^{\prime}$ to the one of the sphere $j$ and the unit vectors $\hat{r}_{j j^{\prime}}^{(j)}$ point from the

origin of the sphere $j$ (as measured in its local coordinate system) to the origin of the sphere $j^{\prime}$. The above expression refers to Dirichlet boundary conditions on the spheres. The case of Neumann boundary conditions (or even mixed boundary conditions) on the spheres follows from the replacement

$$
\frac{j_{l}\left(k a_{j}\right)}{h_{l^{\prime}}^{(1)}\left(k a_{j}\right)} \longrightarrow \frac{\left.\frac{\mathrm{d}}{\mathrm{d} a}\left(a j_{l}(k a)\right)\right|_{a=a_{j}}}{\left.\frac{\mathrm{d}}{\mathrm{d} a^{\prime}}\left(a^{\prime} h_{l^{\prime}}^{(1)}\left(k a^{\prime}\right)\right)\right|_{a^{\prime}=a_{j^{\prime}}}}
$$


etc. A closed-form expression for the two-dimensional case of $n$ disks, corresponding to (15), can be found in [11].

For the case of small scatterers one can even simplify the expression of the multiscattering matrix:

$$
M^{j j^{\prime}}\left(k,\left\{a_{i}\right\},\left\{r_{i j}\right\}\right) \approx \delta^{j j^{\prime}}-\left(1-\delta^{j j^{\prime}}\right) f^{s}\left(k, a_{j}\right) \frac{\mathrm{e}^{\mathrm{i} k r_{j j^{\prime}}}}{r_{j j^{\prime}}}+\mathcal{O}(p \text {-wave }),
$$

since the $s$-wave scattering dominates over all other partial waves. Thus, the non-trivial part of the inverse multi-scattering matrix is given by the propagation of spherical waves modulated by $s$-wave amplitudes $f^{s}\left(k, a_{j}\right)$ between the spheres. From the modified Krein formula the integrated density of states in the case of two small spherical cavities of common radius $a$ and center-to-center separation $r$ can be deduced as [4]

$$
\mathcal{N}_{\mathrm{C}}^{\mathrm{oo}}(k)=-\frac{1}{\pi} \operatorname{Im} \ln \operatorname{det} M^{\mathrm{oo}}(k, a, r) \approx \frac{a^{2}}{\pi r^{2}} \sin [2(r-a) k]+\mathcal{O}\left((k a)^{3}\right) .
$$

This expression should be compared with the semiclassical approximation that sums up all partial waves

$$
\mathcal{N}_{\mathrm{C}, \mathrm{sc}}^{\mathrm{oo}}(k)=\frac{a^{2}}{4 \pi r(r-2 a)} \sin [2(r-2 a) k] .
$$

The latter is the leading contribution to Gutzwiller's trace formula [18], namely the contribution of the non-repeated two-bounce periodic orbit between the two spheres. $S_{\mathrm{po}}(k) / \hbar=2(r-2 a) k$ is the action of the two-bounce periodic orbit, where $2(r-2 a)$ is the length of its geometric path. Note that the semiclassical result is suppressed by a factor of $1 / 2^{2}$ relative to the small-scatterer one. We will see that each factor $1 / 2$ is associated with a semiclassical reflection from one sphere.

It was shown in 4], under the condition $k>1 / a$, that the semiclassical result for $\mathcal{N}_{\mathrm{C}, \mathrm{sc}}^{\text {oo }}(k)$ is a very good approximation of the full quantum-mechanical result calculated from the exact expression (15) of the two-sphere scattering matrix when plugged into the modified Krein formula (12). Therefore, the Casimir energy for two spherical cavities inside a Fermi sea of non-relativistic non-interacting matter modes can be approximated in terms of a spherical Bessel function $j_{1}$ as

$$
\mathcal{E}_{\mathrm{C}}^{\mathrm{oo}}=-\int_{0}^{k_{\mathrm{F}}} \mathrm{d} k \mathcal{N}_{\mathrm{C}}^{o o}(k, a, r) \approx-\frac{k_{\mathrm{F}}^{2}}{2 m_{\mathrm{N}}} \frac{a^{2}}{2 \pi r(r-2 a)} j_{1}\left[2(r-2 a) k_{\mathrm{F}}\right]
$$

where the expression is valid for $k_{\mathrm{F}} a>1$. Here $m_{\mathrm{N}}$ is the mass of the fermionic mode. Note that it is long-ranged, i.e. it scales as $a^{2} / L^{3}$ with $L=r-2 a$. The corresponding fermionic Casimir energy of the sphere-plate system

$$
\mathcal{E}_{\mathrm{C}}^{\mathrm{ol}} \approx-\frac{k_{\mathrm{F}}^{2}}{2 m_{\mathrm{N}}} \frac{a}{2 \pi(r-a)} j_{1}\left[2(r-a) k_{\mathrm{F}}\right]
$$

even scales as $a / L^{2}$ with $L=r-a$. Moreover, the fermionic Casimir energy has in both cases an oscillating behaviour: with increasing distance between the obstacles, the Casimir energy, which starts out to be attractive, will become repulsive, and under a further increase of the distance, it will become attractive again, where the strength 
is of course reduced. This is in contrast to the fixed (negative) sign of the standard Casimir effect with fluctuating electromagnetic or scalar fields between these obstacles. The reason for this oscillating pattern in the fermionic Casimir effect is the presence of a new scale, in addition to the length scale(s), namely the Fermi momentum $k_{\mathrm{F}}$. In fact, the strength of this fermionic Casimir energy is determined by the UV-cutoff of the theory [4]. Also this behaviour distinguishes the fermionic Casimir effect from the standard Casimir effect: the latter is governed by the infrared behaviour of the corresponding density of states.

In [4], the fermionic Casimir energy of the three- and four-sphere system was calculated as well. From the periodic orbit summation it is obvious that there exist genuine three- and more-body interactions. However, it was shown in [4] that the twobounce orbit dominates in the equilateral three- and four-sphere systems. In fact, the billiard analogy holds: it is difficult to make long shots, especially with many bounces - the slightest error ruins the shot. Mathematically, the relative weights calculated from the instabilities of the longer periodic orbits, even weighted with their degeneracy factors, are far less than the weight of the two-bounce orbit. The maximal correction due to the next-to-leading periodic orbit, the triangular three-bounce orbit, is at most $10 \%$ at a distance $r \approx 2.5 a$ and even smaller for other separations.

\section{The sphere-plate case of the scalar Casimir effect}

From now on we will only discuss the scalar Casimir effect, where the fluctuating modes are massless scalar ones. This effect was first investigated by Schaden and Spruch [19, 20] for the case of two spheres subject to Dirichlet boundary conditions in the semiclassical framework of Gutzwiller's trace formula [18]. It was shown that the result of the proximity force approximation emerged from the semiclassical calculation in the limit of vanishing surface-to-surface separation. Gies and coworkers numerically studied the scalar sphere-plate case, again subject to Dirichlet boundary conditions, in the worldline approach [21]. Scardicchio and Jaffe analyzed these results in an optical approach that takes into account not only periodic, but also closed orbits [22].

In the case of two spheres there is a continuous axial symmetry with respect to the line joining the centers of the spheres, and an additional reflection symmetry with respect to any plane containing this (horizontal) symmetry axis. The symmetry group is $C_{\infty h}$ in crystallography group theory notation [23], where the role of the vertical $v$ plane and horizontal $h$ plane is opposite to the one of this reference in order to match the orientation of figure 1. As a consequence of the $C_{\infty h}$ symmetry the inverse multiscattering matrix is separable with respect to the magnetic quantum number $m$ :

$$
\left(\begin{array}{ll}
M^{11} & M^{12} \\
M^{21} & M^{22}
\end{array}\right)_{l m, l^{\prime} m^{\prime}}=\delta_{m m^{\prime}}\left(\begin{array}{cc}
\delta_{l l^{\prime}} & A_{l m, l^{\prime} m}^{12} \\
A_{l m, l^{\prime} m}^{21} & \delta_{l l^{\prime}}
\end{array}\right)
$$

where the indices 1,2 label the two spheres and where $M_{l m, l^{\prime} m^{\prime}}^{j j^{\prime}}$ is the left hand side of (15), whereas $A_{l m, l^{\prime} m^{\prime}}^{j j^{\prime}}$ is the non-trivial term (minus the delta-distribution term 
$\left.\delta^{j j^{\prime}} \delta_{l l^{\prime}} \delta_{m m^{\prime}}\right)$ on the right-hand side of this equation - both adjusted to the two-sphere case.

The case of two identical spheres at a center-to-center distance $r$ contains the case of a sphere and a plate at a center-to-plate distance $R=r / 2$ as can be seen in figure 1. Because of the additional two-fold reflection symmetry with respect to the vertical

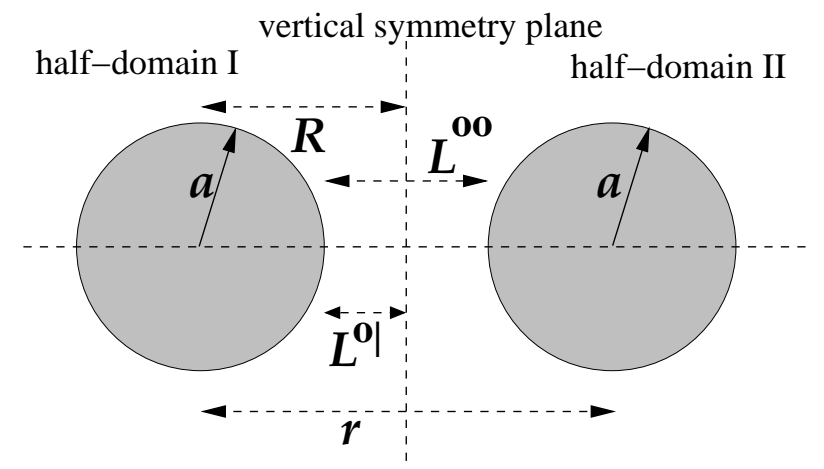

Figure 1. Two spheres of common radius $a$ at a center-to-center separation $r$. The vertical symmetry plane splits the full domain into two half-domains I and II. The center-to-plane separation $R$, the surface-to-surface separation $L^{00}$ in the full domain and $L^{0 \mid}$ in the half-domain are indicated as well.

symmetry plane, the symmetry group of the two-sphere case with common radius $a$ is even $D_{\infty v}$. Therefore, the global domain splits into two half-domains (called I and II) that are separated by the vertical symmetry plane. Moreover, all scattering waves can be separated into symmetric ones (subject to Neumann boundary conditions) and antisymmetric ones (subject to Dirichlet boundary conditions) with respect to this plane. Thus there exist two classes of inverse multi-scattering matrices in one half-domain, one subject to Neumann boundary conditions $(\mathrm{N})$ and one subject to Dirichlet boundary conditions (D) on the vertical symmetry plan [6]:

$$
\left.M_{l l^{\prime}}^{\mathrm{oo}(m)}\right|_{\mathrm{N}}=\delta_{l l^{\prime}}+A_{l l^{\prime}}^{(m)},\left.\quad M_{l l^{\prime}}^{\mathrm{oo}(m)}\right|_{\mathrm{D}}=\delta_{l l^{\prime}}-A_{l l^{\prime}}^{(m)}
$$

(using the short-hand notation $A_{l l^{\prime}}^{(m)} \equiv A_{l m, l^{\prime} m}^{12}=A_{l m, l^{\prime} m}^{21}$ ), and the associated determinants factorize as

$$
\begin{aligned}
\operatorname{det} M^{\mathrm{oo}}(k, a, r) & =\prod_{m=-\infty}^{\infty} \operatorname{det} M^{\mathrm{oo}(m)}(k, a, r) \\
& =\left.\left.\operatorname{det} M^{\mathrm{oo}}(k, a, r)\right|_{\mathrm{N}} \operatorname{det} M^{\mathrm{oo}}(k, a, r)\right|_{\mathrm{D}} .
\end{aligned}
$$

Therefore the determinant for the sphere-plate case under Dirichlet boundary conditions can be inferred from $\left.\operatorname{det} M^{\circ o}(k, a, r)\right|_{\mathrm{D}}$ as follows:

$$
\operatorname{det} M^{\mathrm{ol}}\left(k, a, L^{\mathrm{o} \mid}\right)=\left.\operatorname{det} M^{\mathrm{oo}}\left(k, a, r=2\left(L^{\mathrm{o} \mid}+a\right)\right)\right|_{\mathrm{D}}
$$

and the pertinent Casimir energy is given as [6]

$$
\mathcal{E}_{\mathrm{C}}^{\mathrm{o}}(a, L)=\left.\frac{\hbar c}{2 \pi} \int_{0}^{\infty} \mathrm{d} k_{4} \ln \operatorname{det} M^{\mathrm{oo}}\left(\mathrm{i} k_{4}, a, r=2(L+a)\right)\right|_{\mathrm{D}} .
$$


The exact data generated by this equation are reported in [6]. They are compatible with the numerical data of the worldline approach [21] between $L=a / 8$ and $L=a$ if the statistical error bars of the worldline data are taken into account, see [6]. Moreover, the (systematically and statistically improved) worldline data of [24, 25], which extend up to $L=16 a$ and up to $L<100 a$, respectively, do nicely agree with the exact data of [6]. In principle, the calculation based on (26) is exact and applicable for all separations of the obstacles. In practice, it is more suited for large- to medium-range separations. The reason is that the Casimir energy for the scalar case is dominated by momenta $k \sim 1 / L$ where $L$ is the separation scale. As shown in [6] for the scalar sphere-plate case, the Casimir integration can be truncated at $k_{\max } \sim 10 / L$ corresponding to a truncation in the angular momentum space at $l_{\max } \leq(\mathrm{e} / 2) k_{\max } a \approx 14 a / L$. For $L<0.1 a$ the matrices increase rapidly in size and the angular-momentum algebra (see the $3 \mathrm{j}$ symbols in (15d) becomes very cumbersome.

Let us rather concentrate on the asymptotical behaviour (surface-to-surface separation $L \gg a$ ) of Casimir energy given by (26). The $s$-wave approximation is not needed for the fermionic Casimir energy since it is governed by the UV part of the density of states (i.e. by the contribution at the Fermi momentum $k_{\mathrm{F}}$, assuming $\left.k_{\mathrm{F}}>1 / a\right)$. However, it dominates the asymptotics of the fluctuating-scalar Casimir effect at very large separations $(L \gg a)$, as this Casimir-energy type is governed by the infrared behaviour of the density of states at $k \sim 1 / L$ or less. The Casimir energy of the sphere-plate case subject to Dirichlet boundary conditions is given at large separations $L \gg a$ (in the $s$-wave approximation) as [6]

$$
\begin{aligned}
\mathcal{E}(L) & \sim-\frac{\pi^{3} \hbar c a}{1440 L^{2}} \frac{90}{\pi^{4}} \frac{2}{(1+a / L)(1+a / 2 L)} \\
& \rightarrow-\frac{\pi^{3} \hbar c a}{1440 L^{2}} \frac{90}{\pi^{4}} \times 2=-\frac{\pi^{3} \hbar c a}{1440 L^{2}} \times 1.847 \cdots
\end{aligned}
$$

As in section 4 there is a relative factor of 2 originally found in [4] between the $s$-wave and semiclassical result for each reflection off a sphere. This applies also for the fluctuatingscalar Casimir effect, if the relativistic dispersion $E=\hbar c k$ and the suppression of the repeats of the semiclassical two-bounce orbit at large separations (by the removal of the term $\left.\sum_{n=1} n^{-4}=\pi^{4} / 90\right)$ are taken into account [6]. Therefore, the exact result of the fluctuating-scalar Casimir energy for the very far separated Dirichlet sphere-plate system is enhanced by a factor of 2 relative to the semiclassical result and by a factor of $2 \times\left(90 / \pi^{4}\right)$ relative to the leading term $-\pi^{3} \hbar c a /\left(1440 L^{2}\right)$ of the proximity-force approximation (PFA) [3]. The leading term of the PFA, in other words the PFA at vanishing distances, where the repeats of the two-bounce orbit cannot be neglected and add up to the factor $\pi^{4} / 90$, was - as mentioned - confirmed semiclassically in [19]. In this connection, it should be noted that the result quoted in [6] for the semiclassical approximation to scalar Dirichlet sphere-plate case

$$
\mathcal{E}_{\mathrm{sc}}^{\mathrm{o}}(a, L)=-\frac{\hbar c}{16 \pi} \frac{a}{L^{2}}\left(\frac{\pi^{4}}{90}\right)\left[1-\left(\frac{5}{\pi^{2}}-\frac{1}{3}\right) \frac{L}{a}+\mathcal{O}\left([L / a]^{2}\right)\right]
$$


also applies for the scalar Neumann case, since both semiclassical calculations differ only by the Maslov indices (i.e by a minus sign for each reflection from a Dirichlet surface) and since the number of reflections is even for a self-retracing orbit. The electromagnetic case in the semiclassical approximation is then given by the sum of both scalar cases. It is therefore twice as big as (28) and the first correction to the leading PFA result is predicted to be negative.

In the case of the electromagnetic Casimir effect for the sphere-plate system, the $s$ wave dominance at large separation has to be replaced by a $p$-wave dominance, since the charge-neutrality of the sphere forbids a monopole term, whereas the standard CasimirPolder energy is dominated by induced-dipole contributions. If one removes by hand the $s$-wave contribution from the Casimir energy of the scalar Dirichlet sphere-plate system, the remaining energy is dominated at large separations by the $p$-wave contribution

$$
\mathcal{E}_{\text {p-wave }}(L) \sim-\frac{5 \pi^{3} \hbar c a^{3}}{1440 L^{4}} \frac{90}{\pi^{4}} .
$$

Note that this expression is compatible with the $a^{3} / L^{4}$ scaling of the Casimir-Polder energy for a molecule-plate system [26], but the prefactor is different [27].

Together with M Bordag [28] the asymptotics of the Dirichlet sphere-plate problem was worked out to sixth subleading order in the ratio of the sphere radius $a$ and the center-to-plate separation $R=r / 2$ :

$$
\begin{gathered}
E_{\mathrm{D}, l \geq 0}=-\frac{\hbar c a}{8 \pi R^{2}}\left\{1+\frac{5}{8} \frac{a}{R}+\frac{421}{144}\left(\frac{a}{R}\right)^{2}+\frac{535}{1152}\left(\frac{a}{R}\right)^{3}+\frac{3083041}{518400}\left(\frac{a}{R}\right)^{4}\right. \\
\left.-\frac{2741117}{1382400}\left(\frac{a}{R}\right)^{5}+\frac{557222415727}{36578304000}\left(\frac{a}{R}\right)^{6}+\cdots\right\}
\end{gathered}
$$

Even higher-order contributions (up to ninth order) were considered in this asymptotic series, but did not lead to any improvements. For practical purposes the sixth order expression is still useful down to separations $L=0.7 a$ ( or $R=1.7 a$ ), see figure 2. If the $s$-wave contribution is removed by hand, the corresponding expansion reads [28]

$$
\begin{array}{r}
E_{\mathrm{D}, l>0}=-\frac{5 a^{3}}{16 \pi R^{4}}\left\{1+\frac{56}{25}\left(\frac{a}{R}\right)^{2}-\frac{597}{640}\left(\frac{a}{R}\right)^{3}+\frac{10453}{1750}\left(\frac{a}{R}\right)^{4}\right. \\
\left.-\frac{16557}{1600}\left(\frac{a}{R}\right)^{5}+\frac{394844679647}{9144576000}\left(\frac{a}{R}\right)^{6}+\cdots\right\} .
\end{array}
$$

Finally, for the corresponding Neumann sphere-plate system the removal of the $s$-wave contribution is natural and the corresponding result is given by [28]

$$
\begin{aligned}
E_{\mathrm{N}, l>0}=- & \frac{10 a^{3}}{16 \pi R^{4}}\left\{1+\frac{63}{100}\left(\frac{a}{R}\right)^{2}+\frac{597}{320}\left(\frac{a}{R}\right)^{3}-\frac{4159}{14000}\left(\frac{a}{R}\right)^{4}\right. \\
& \left.-\frac{271437}{25600}\left(\frac{a}{R}\right)^{5}+\frac{148355331834}{2286144000}\left(\frac{a}{R}\right)^{6}+\cdots\right\} .
\end{aligned}
$$

For reasons of completeness, we also report the numerical result of the pure $s$-wave contribution for the sphere-plate case under Neumann boundary conditions, although it is of course artificial:

$$
E_{\mathrm{N}, l=0}=-\frac{1}{4 \pi R} \times 0.46066 \ldots \times\left\{1+\mathcal{O}\left(\frac{a}{R}\right)\right\} .
$$




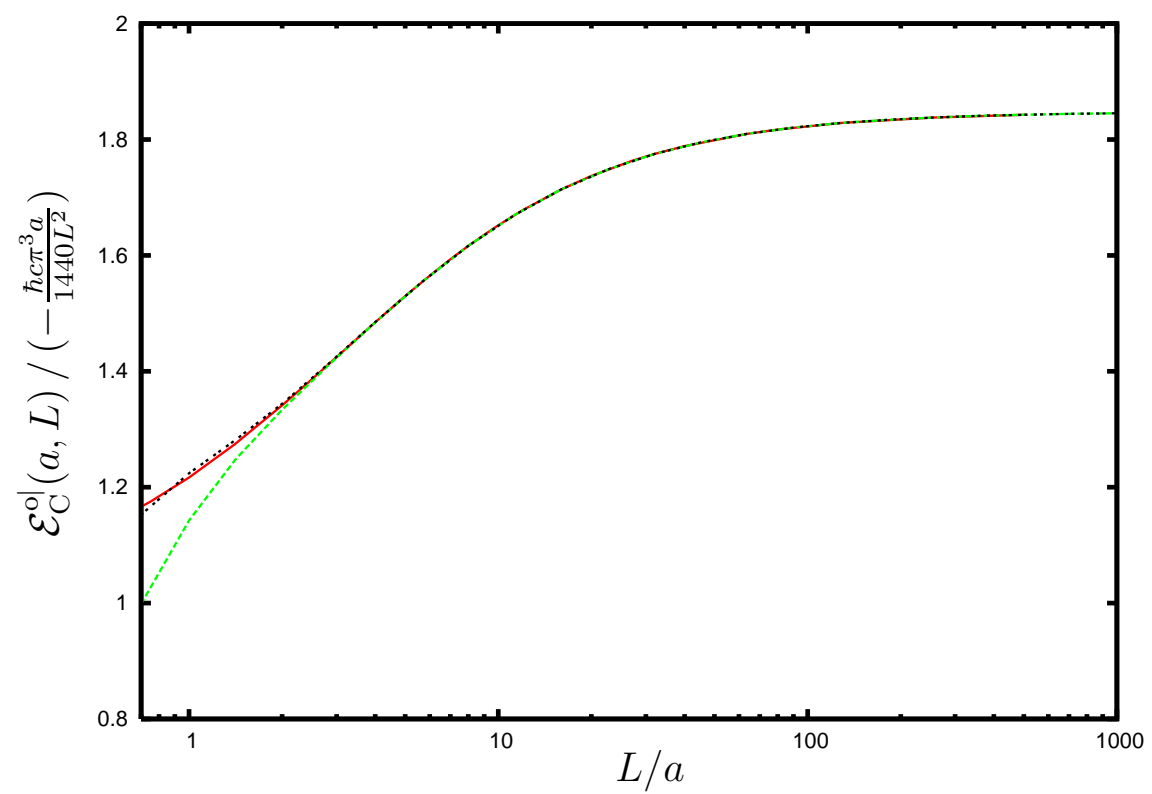

Figure 2. Exact and asymptotic results for the scalar Casimir energy of the sphereplate configuration with Dirichlet boundary conditions are shown shown in units of $-\hbar c \pi^{3} a /\left(1440 L^{2}\right)$ as function of the ratio of the surface-to-surface-separation $L$ and the sphere radius $a$. The exact result from (26) is plotted as full line (in red), the asymptotic approximation (30) up to the $(a / R)^{4}$ term as dashed line (in green) and up to the $(a / R)^{6}$ term as short-dashed line (in blue).

Note that in this case the infrared limit $k_{4} \rightarrow 0$ and the asymptotic limit $R / a \rightarrow \infty$ do not commute in (26).

Finally, for comparison, the result of the Casimir-Polder calculation in the limit of a perfectly conducting sphere as calculated in [27] is listed here as well:

$$
E_{\mathrm{EM}, l>0}=-\frac{(3+6) a^{3}}{16 \pi R^{4}} \times\left\{1+\mathcal{O}\left(\frac{a}{R}\right)\right\} .
$$

We see that the relative ratio of the leading Dirichlet and Neumann $l>0$ contributions is correct, but that the scalar result is bigger than the electromagnetic one by a factor of $5 / 3$.

\section{Final remarks}

In the meantime, after the work [6] appeared, further exact Casimir results for nonseparable systems have been reported. In [29], the Casimir interaction between a plate and a cylinder was calculated for the background of a fluctuating electromagnetic field. The exact zero-point interaction between two non-concentric cylinders was studied in [30]. The Casimir effect for a sphere and a cylinder in front of a plane and corrections to the proximity force theorem were investigated in [31. The Casimir forces between arbitrary compact objects were investigated in [32]; see also the study [33] in the $T$ operator approach and [34, 35]. 
Finally, let me mention the way in which the Casimir energy per unit length for $n$ non-overlapping parallel cylinders of infinite length can be derived from the modified Krein formula of the two-dimensional $n$-disk case. For a cut perpendicular to the cylinder axes the modified Krein formula of the $n$-disk case can be applied, provided the wave number $k$ in that formula is replaced by the modulus of the perpendicular wave number $k_{\perp}$ (the wave number along the cut). Moreover, the phase space integration $L_{\|} \int \mathrm{d} k_{\|} /(2 \pi)$ along the parallel direction to the cylinder axes has to be added and the resulting integrals have to be weighted by the correct energy-dispersion $\hbar c \sqrt{k_{\|}^{2}+k_{\perp}^{2}}$. The corresponding Casimir energy per unit length therefore reads

$$
\begin{aligned}
\mathcal{E}_{\mathrm{C}} / L_{\|} & =\int_{-\infty}^{\infty} \frac{\mathrm{d} k_{\|}}{2 \pi} \int_{0}^{\infty} \mathrm{d} k_{\perp} \frac{1}{2} \hbar c \sqrt{k_{\|}^{2}+k_{\perp}^{2}} \delta \rho_{\mathrm{C}}\left(k_{\perp}, n \text {-disk }\right) \\
& =\int_{-\infty}^{\infty} \frac{\mathrm{d} k_{\|}}{2 \pi} \int_{0}^{\infty} \mathrm{d} k_{\perp} \frac{1}{2} \hbar c \sqrt{k_{\|}^{2}+k_{\perp}^{2}} \frac{d}{d k_{\perp}} \frac{-1}{\pi} \operatorname{Im} \ln \operatorname{det} M\left(k_{\perp}, n \text {-disk }\right) \\
& =\frac{\hbar c}{2 \pi^{2}} \int_{-\infty}^{\infty} \mathrm{d} k_{\|} \int_{\left|k_{\|}\right|}^{\infty} \mathrm{d} k_{4} \frac{k_{4}}{\sqrt{k_{4}^{2}-k_{\|}^{2}}} \ln \operatorname{det} M\left(\mathrm{i} k_{4}, n \text {-disk }\right) \\
& =\frac{\hbar c}{4 \pi} \int_{0}^{\infty} \mathrm{d} k_{4} k_{4} \ln \operatorname{det} M\left(\mathrm{i} k_{4}, n \text {-disk }\right)
\end{aligned}
$$

\section{Summary}

We have shown that the Casimir energy can be re-defined as the vacuum energy of the geometry-dependent part of the density of states and that the latter can be calculated form the multi-scattering phase shift of a modified Krein formula. The nonoverlapping, in general non-separable $n$-sphere, sphere-plate, $n$-disk (and $n$-cylinder) Casimir problems can be solved exactly in the scalar (and also in the fermionic) case. The calculation is not plagued by the subtraction of single-sphere contributions or by the removal of diverging ultraviolet contributions. All involved determinants exist and

are finite since the pertinent T-matrices are trace-class. The large-distance behaviour is dominated by the $s$-wave scattering in the case of the scalar Casimir effect with Dirichlet boundary conditions and by the $p$-scattering for the corresponding Neumann case as in the electromagnetic scenario. The Dirichlet or Neumann boundary conditions can even be replaced by mixed boundary conditions. The presented method can easily be applied to any number of spheres or cylinders with or without planes (in two dimensions: disks with or without lines). Moreover, the spheres (or disks) can be replaced by other objects or even smooth potentials or non-ideal reflector, as long as these objects do not overlap. The finite surface thicknesses can be booked as Weyl-term contributions.

\section{Acknowledgments}

A.W. would like to thank the organizer Michael Bordag for the invitation to this excellent workshop QFEXT'07 in Leipzig. 


\section{References}

[1] Casimir H B G 1948 Proc. Kon. Ned. Akad. Wetensch. 51793

[2] Bordag M, Mohideen U and Mostepanenko V M 2001 Phys. Rept. 3531

[3] Derjaguin B V, Abrikosova I I and Lifshitz E M 1956 Quart. Rev. 10 295; Blocki J, Randrup J, Swiateck W J and Tsang C F 1977 Ann. Phys., NY 105427

[4] Bulgac A and Wirzba A 2001 Phys. Rev. Lett. 87120404 arXiv:nucl-th/0102018

[5] Bulgac A, Magierski P and Wirzba A 2005 Europhys. Lett. 72327 arXiv:cond-mat/0406255

[6] Bulgac A, Magierski P and Wirzba A 2006 Phys. Rev. D 73025007 arXiv:hep-th/0511056

[7] Wirzba A, Bulgac A and Magierski P 2006 J. Phys. A: Math. Gen. 396815 arXiv:quant-ph/0511057

[8] Krein M G 1953 Mat. Sborn. (N.S.) 33 597; Krein M G 1962 Sov. Math.-Dokl. 3 707; Birman M Sh and Krein M G 1962 Sov. Math.-Dokl. 3740

[9] Beth E and Uhlenbeck G E 1937 Physica 4 915; Huang K 1987 Statistical Mechanics, John Wiley \& Sons, New York, ch. 10.3; Friedel J 1958 Nuovo Cim. Ser. 10 Suppl. 7287

[10] Gaspard P and Rice S A 1989 J. Chem. Phys. 90 2225; 90 2242; 902255

[11] Wirzba A 1999 Phys. Rept. 3091 arXiv:chao-dyn/9712015

[12] Wirzba A and Henseler M 1998 J. Phys. A: Math. Gen. 312155 arXiv:chao-dyn/9702004

[13] Henseler M, Wirzba A and Guhr T 1997 Ann. Phys., NY 258286 arXiv:chao-dyn/9701018

[14] Eckhardt B 1987 J. Phys. A: Math. Gen. 205971

[15] Cvitanović P and Eckhardt B 1989 Phys. Rev. Lett. 63823

[16] Wirzba A 1992 CHAOS 277

[17] Wirzba A 1993 Nucl. Phys. A 560136

[18] Gutzwiller M C 1990 Chaos in Classical and Quantum Mechanics (Springer, New York)

[19] Schaden M and Spruch L 1998 Phys. Rev. A 58935

[20] Schaden M and Spruch L 2000 Phys. Rev. Lett. 84459

[21] Gies H, Langfeld K and Moyaerts L 2003 J. High Energy Phys. 06018 arXiv:hep-th/0303264

[22] Jaffe R L and Scardicchio A 2004 Phys. Rev. Lett. 92 070402; Scardicchio A and Jaffe R L 2005 Nucl. Phys. B $\mathbf{7 0 4} 552$

[23] Hamermesh M 1962, Group Theory and Its Applications to Physical Problems (Addison-Wesley, Reading, MA), Chap. 9-1

[24] Gies H and Klingmüller K 2006 J. Phys. A: Math. Gen. 396415 arXiv:hep-th/0511092

[25] Gies H and Klingmüller K 2006 Phys. Rev. D 74045002 arXiv:quant-ph/0605141

[26] Casimir H B G and Polder D 1948 Phys. Rev. 73360

[27] Datta T and Ford L H 1981 Phys. Lett. 83A 314

[28] Bordag $\mathrm{M}$ and Wirzba A unpublished

[29] Emig T, Jaffe R L, Kardar M and Scardicchio A 2006 Phys. Rev. Lett. 96080403 arXiv:cond-mat/0601055

[30] Mazzitelli F D, Dalvit D A R and Lombardo F C 2006 New J. of Phys. 8240 arXiv:quant-ph/0610181

[31] Bordag M 2006 Phys. Rev. D 73125018 arXiv:hep-th/0602295]

[32] Emig T, Graham N, Jaffe R L and Kardar M 2007 arXiv:0707.1862 [cond-mat.stat-mech] and arXiv:0710.3084 [cond-mat.stat-mech]

[33] Kenneth O and Klich I 2007 arXiv:0707.4017 [quant-ph]

[34] Emig T and Jaffe R L 2007 arXiv:0710.5104 [quant-ph]

[35] Milton K A and Wagner J 2007 arXiv:0711.0774 [hep-th] 